\title{
Síndrome de Lemièrre. Descripción de dos nuevos casos y revisión de la literatura
}

\author{
J. L. FERNÁNDEZ REYES, J. J. DONES CARVAJAL, I. Ma. CARCELÉN MORA, \\ J. Ma . GUTIÉRREZ MORENO, ${ }^{\mathrm{a}}$. C. LEA PEREIRA, $\mathrm{M}^{\mathrm{a}}$. A. CAMACHO PASTOR* \\ Servicio de Medicina Interna, sección C. Hospital General de Especialidades "Ciudad de \\ Jaén". *Medicina Familiar y Comunitaria. Jaén
}

\author{
LEMIĖRRE'S SYNDROME. DESCRIPTION OF TWO NEW CASES \\ AND BIBLIOGRAPHYC REVIEW
}

\begin{abstract}
RESUMEN
La tromboflebitis séptica de la vena yugular interna como complicación de las infecciones orofaríngeas, tras un cuadro de sepsis postangina, fue descrita por primera vez a principios de siglo por Lemièrre. Para el diagnóstico de este síndrome, se requiere la existencia de abscesos metastásicos a distancia. Estos se localizan más frecuentemente a nivel pulmonar, pero también se han descrito en tejidos blandos. En este trabajo presentamos dos nuevos casos de este infrecuente síndrome, observados en el período de un año. En nuestro conocimiento, no han sido descritos anteriormente abscesos metastásicos hepáticos en este cuadro, que observamos en uno de nuestros pacientes. También aportamos nuestra experiencia en el tratamiento con heparina de bajo peso molecular de la tromboflebitis venosa yugular, que forma parte de este síndrome.
\end{abstract}

PALABRAS CLAVE: Síndrome de Lemièrre.

\begin{abstract}
Septic thrombophlebitis of internal jugular vein following orop haryngeal infections, referred to as postanginal sepsis, was first descri bed at the begining of the century by Lemièrre. For diagnostic of Lemiè rre's syndrome, distant metastatic abscesses from septic embolization are required. These are placed most frequently in the lungs, but also ocu rred in soft tissues. Two cases of this inusual syndrome seen in a year period, are reported in this brief communication. We described the first liver metastatic infection, in the syndrome. Successful result was obser ved when we use a low molecular weight heparin as treatment in the sep tic thrombophlebitis of internal jugular vein.
\end{abstract}

KEY WORDS: Lemièrre`s syndrome.

Fernández Reyes JL, Dones Carvajal JJ, Carcelén Mora IM ${ }^{a}$, Gutiérrez Moreno JM ${ }^{a}$, Lea Pereira M ${ }^{a}$ C, Camacho Pastor Má Aíndrome de Lemièrre. Descripción de dos nuevos casos y revisión de la literatura. An Med Interna (Madrid) 2002; $19: 139-142$.

\section{INTRODUCCIÓN}

Fue en 1936 cuando A. Lemièrre describió por primera vez un grave cuadro clínico (1) caracterizado por la existencia de infección nasofaríngea, sobre todo abscesos amigdalares y periamigdalares, lesiones en boca y mandíbula en relación con otitis media y mastoiditis, acompañado de tromboflebitis de la vena yugular interna (2-5) y metástasis sépticas pulmonares (sepsis postangina), que posteriormente también fueron descritas por diversos autores a distintos niveles, sobre todo pulmonar (4,6-8) (entre un $89 \%$ y un $97 \%(2,3,9-13)$ de los casos), y con menor frecuencia en partes blandas $(2,14)$, meninges $(2,9)$, sistema nervioso central (11), bazo (15), tejido muscular (14), articulaciones $(16,17)$ y tejido óseo (17). En el $80 \%$ de los casos está implicado el Fusobacterium necrop horum $(2,3,11,16)$, conocido hasta los años setenta como bacillus fundiliformis, un bacilo anaerobio no esporulado gram negativo, que es aislado en los hemocultivos de estos pacien- tes, aunque frecuentemente la infección es polimicrobiana $(2,3,9,12)$. Desde el comienzo de la era antibiótica, este síndrome que antes era considerado letal hasta en un $90 \%$ de los casos (9), es menos frecuente.

Se presentan dos nuevos casos de este síndrome atendidos en los últimos dos años en nuestro Servicio, con revisión de la literatura (MEDLINE, IME, período 1976Noviembre 1998, palabra clave: Lemièrre), comentando especialmente los aspectos clínicos, diagnósticos y terapéuticos, con objeto de facilitar su conocimiento y el diagnóstico de futuros nuevos casos. Así mismo, queremos puntualizar la presentación en uno de nuestros pacientes de metástasis sépticas hepáticas, que no hemos encontrado descritas en anteriores trabajos formando parte de este síndrome, y comentar nuestra experiencia en el tratamiento de la trombosis venosa yugular con heparina de bajo peso molecular, que no ha sido utilizada previamente en esta indicación por otros autores.

Trabajo aceptado: 14 de septiembre de 2000

Correspondencia: $\mathrm{M}^{\mathrm{a}}$ de los Angeles Camacho Pastor. C/ Fuente del Alamillo nº 7, portal 4, $3^{\circ}$ Al. 23006. Jaén. 


\section{CASOS APORTADOS}

Caso 1. Mujer de 18 años que dos semanas antes de su ingreso había presentado un cuadro de faringoamigdalitis aguda con fiebre de $38^{\circ} \mathrm{C}$. Había realizado tratamiento con paracetamol sin demandar asistencia. En el momento de su ingreso había remitido la sintomatología a nivel orofaríngeo, pero persistía la fiebre, junto a tos seca y dolor costal derecho, añadiéndose en los dos últimos días dolor en región laterocervical izquierda, donde presentaba adenopatías dolorosas y tumefacción local, junto a amígdala izquierda hipertrófica. En la analítica destacaba: leucocitos 29x109/L (89\% PMN), Hb 10,8 g/dl, Fosfatasa alcalina $256 \mathrm{U} / \mathrm{L}$, GGT $88 \mathrm{U} / \mathrm{L}$, fibrinógeno 6,8 g/l, VSG $1^{\text {a }}$ hora $84 \mathrm{~mm}$; el resto de la bioquímica completa, proteinograma, complemento, ANA, anti DNA, anti ENA y estudio de coagulación era normal. Los hemocultivos, utilizando sistema Bact-Alert (Organon Teknika) con identificación por sistema VITEK y antibiograma por sistema disco-placa, fueron positivos para Porphyromona asaccharolytica. La radiología de tórax mostraba una imagen nodular en lóbulo medio y otra basal derecha. El ecocardiograma fue normal. La TAC de cuello mostraba una tumoración en espacio parafaríngeo izquierdo que se extendía desde región amigdalar epiglótica, compatible con proceso inflamatorio no organizado, y una zona hipodensa central a nivel de vena yugular interna izquierda. En Eco-Doppler se confirmó la existencia de una vena yugular interna izquierda trombosada. Se indicó tratamiento con penicilina $\mathrm{G}$ sódica, y ante la persistencia de la fiebre pasados tres días, se indicó anticoagulación con heparina de bajo peso molecular, desapareciendo la fiebre y la sintomatología local inflamatoria, así como la imagen nodular en la radiografía de tórax de control realizada a los 7 días de iniciar el tratamiento antibiótico. A las 4 semanas de ingreso fue dada de alta con tratamiento antibiótico por vía oral (amoxicilina $1 \mathrm{~g}$ cada 8 horas durante 3 semanas) y acenocumarol que mantuvo durante 3 meses. Pasados 6 meses, la paciente está asintomática y en la imagen de resonancia magnética se apreciaba una disminución de calibre de vena yugular interna izquierda.

Caso 2. Varón de 26 años que consulta por fiebre de 2 semanas de evolución. En los primeros cinco días del cuadro había presentado molestias faringoamigdalares, y había realizado tratamiento con roxitromicina. A su ingreso, salvo ligero enrojecimiento faríngeo, el resto de la exploración física completa era normal. Pasadas 24 horas, al explorar de nuevo al paciente, se apreciaba dolorimiento a la palpación de hipocondrio derecho. Se realizó TAC abdominal donde apareció lesión ocupante de espacio hipodensa y parcialmente septada en segmento posterior de lóbulo hepático derecho (Fig. 1), procediéndose a drenaje quirúrgico dirigido por TAC. El cuadro clínico empeoró en las horas siguientes, presentando criterios de shock séptico con coagulopatía de consumo, que hizo necesario su ingreso en UCI, donde precisó apoyo respiratorio con ventilación asistida por cuadro de distrés respiratorio, y medicación vasoactiva, iniciándose tratamiento antibiótico con imipenem y ornidazol. Tras superar esta situación, fue dado de alta en UCI a los 12 días. En hemocultivos y en drenaje de absceso hepático, que requirió en tres ocasiones, utilizando sistema Bact-Alert (Organon Teknika) con identificación por sistema VITEK y antibiograma por sistema disco-placa, se aisló Fusobacterium varium. El estudio de inmunidad celular y humoral fue normal. Tras una semana de alta de UCI en que permaneció afebril y tras completar tres semanas de antibioterapia, presentó de nue vo fiebre y tumefacción en zona paracervical y hueco supraclavicular derecha, confirmándose el diagnóstico clínico de sospecha de trombosis venosa profunda de vena yugular interna mediante resonancia magnética, tras lo que se prosiguió igual tratamiento antibiótico y se indicó tratamiento anticoagulante con heparina sódica. La fiebre remitió en 48 horas y el paciente fue dado de alta asintomático a las 4 semanas, con acenocumarol durante 3 meses, y metronidazol oral (500 mg cada 8 horas) y amoxicilina ( 1 g cada 8 horas) durante 21 días. En revisión pasados 2 meses, el paciente estaba asintomático, la TAC mostraba ausencia de lesiones ocupantes de espacio en hígado,

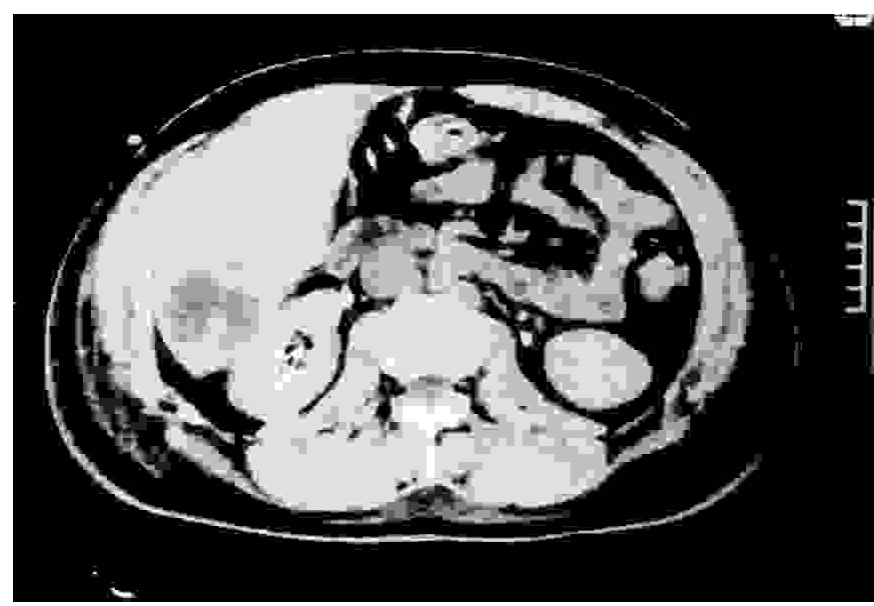

Fig. 1. TAC abdominal. Se aprecian las lesiones hipodensas en parénquima hepático correspondientes al absceso a este nivel.

y la resonancia magnética de cuello mostraba ligera disminución de calibre de vena yugular interna derecha.

\section{DISCUSIÓN}

El síndrome de Lemièrre (sepsis postangina), se caracteriza por la presencia de tromboflebitis séptica de la vena yugular interna junto a abscesos metastásicos a diversos niveles, sobre todo pulmonares. Este cuadro es más frecuente en adultos jóvenes previamente sanos, sin déficit inmunitario, hábitos tóxicos ni otros factores de riesgo predisponentes $(5,9,16,18)$. Existen algunas publicaciones en las que se describe un síndrome que reúne características clínicas suficientes para ser diagnosticado de sepsis postangina, y no se hace referencia a él como tal (19), hecho que ha conducido a algunos autores a calificar este cuadro como la "enfermedad olvidada" ("irreconocida", diríamos nosotros). Aunque algunos autores requieren la existencia previa de un cuadro amigdalar grave (angina de Plaut-Vincent) (20), la mayoría de los casos de este síndrome se han descrito después de episodios subclínicos de infección orofaríngea aguda $(19,21)$, ocurridos entre varios días y dos semanas antes de la diseminación séptica hematógena (18), como ocurrió en nuestros dos pacientes, que incluso no demandaron asistencia médica durante la evolución de su cuadro orofaríngeo agudo. También se ha descrito este síndrome como complicación secundaria a manipulaciones dentarias $(3,22)$, osteítis peridental (19), sinusitis (23), mastoiditis (24) y tras la presentación de un cuadro clínico con confirmación serológica de mononucleosis infecciosa (23).

Las metástasis sépticas se han descrito a distintos niveles, sobre todo pulmonar $(4,6-8)$ (entre un $89 \%$ y un $97 \%(2,3,9$ 13) de los casos), en partes blandas $(2,14)$, meninges $(2,9)$, sistema nervioso central (11), bazo (15), tejido muscular (14), articulaciones $(16,17)$ y tejido óseo (17). Aunque se describen alteraciones en la función hepática (aumento moderado de fosfatasa alcalina, gammaglutamiltranspeptidasa y transaminasas (41), en relación con una colestasis simple, con mínimos infiltrados de células plasmáticas y linfocitos en los conductos biliares e hiperplasia de las células de Küpffer, no hemos encontrado en la literatura revisada referencia a localización hepática de las metástasis sépticas en este síndrome, como ocurrió en el caso 2. 
Se relaciona con bacteriemia por anaerobios, habiéndose descrito asociado a Fusobacterium necrophorum y Porphyromona asaccharolytica (3), no siendo infrecuente su origen polimicrobiano (3). Otros gérmenes propios de la cavidad oral que se han relacionado con la sepsis postangina son Eikenella corrodens (42) y Estreptococos beta-hemolíticos. Fusobacterium varium, flora habitual del tracto respiratorio superior, cavidad oral e intestino (25), también ha sido descrito como causante de mastoiditis (26), artritis séptica (27) e infección intraabdominal (28) y utilizado como modelo experimental de sepsis intraabdominal en ratas (29). Porphyromona assacharolytica (Bacteroides assacharolytica en la antigua clasificación (25) ) se ha aislado mediante cromatografía de gases $(30,31)$ en abscesos pulmonares (32), infecciones dentales (32) y sinusitis crónica en niños (32); forma parte de la flora habitual en igual localización que Fusobacterium varium (25) $\mathrm{y}$, además es hallado en genitales externos, uretra, vagina y endocérvix (25).

La trombosis de vena yugular fue diagnosticada en nuestros dos pacientes por TAC (33) y Eco-Doppler en el caso 1 y por RMN en el caso 2, técnicas de reconocido valor por otros autores para el diagnóstico de trombosis venosa yugular $(4,7,18,34,35)$. Es recomendable realizar alguna de estas técnicas de imagen ante la existencia de bacteriemia por Fusobacterium (7), o bacteriemia por otro anaerobio, aún en ausencia de clínica de trombosis a dicho nivel, dado que los signo orofaríngeos y cervicales pueden estar ausentes (36) o sólo ser discretos en forma de tumefacción o induración a nivel cervical (9). Algunos autores han señalado la necesidad de realizar hemocultivos y cuidadosa exploración de cuello a los pacientes con faringoamigdalitis severa (37), para evitar el retraso o incluso la omisión diagnóstica de esta entidad.

Se han descrito graves complicaciones de este síndrome, como distrés respiratorio (38), shock séptico (23), coagulopa- tía de consumo (23) (como en nuestro segundo caso) y meningitis (23). Para la resolución del cuadro es clave, como ocurrió en uno de nuestros casos, sumar al tratamiento antibiótico, que debe ser intravenoso, a altas dosis (4) y prolongado durante varias semanas, el drenaje quirúrgico de los abscesos $(9,11,18)$. El antibiótico de elección es la penicilina $\mathrm{G}$ asociada con clindamicina, metronidazol, cloranfenicol o cefoxitina $(2,3,9,12,21)$. El uso de anticoagulación en estos pacientes es controvertido $(2,10)$, habiéndose observado una importante mejoría con su utilización en los casos en los que no se observa una respuesta adecuada al tratamiento antibiótico (12), se produce recurrencia de la fiebre o se presenta durante la evolución nuevos fenómenos embólicos a pesar del tratamiento antibiótico. Ya existen evidencias del efecto beneficioso obtenido con la anticoagulación en otras formas de tromboflebitis séptica de grandes vasos (39). En nuestra experiencia (un paciente fue tratado con heparina de bajo peso molecular y otro con heparina sódica -INR entre 2 y 3-, y ambos posteriormente con acenocumarol durante 3 meses), la anticoagulación ayudó a la resolución del cuadro, hecho que ha sido observado por otros autores $(10,39)$. A veces, en caso de persistencia del estado séptico, y si se confirma la ausencia de retorno venoso yugular tras el tratamiento arriba indicado, puede estar indicada la ligadura de vena yugular $(4,36)$, aunque este recurso terapéutico rara vez es necesario.

Pensamos que el diagnóstico de este síndrome debe ser sospechado ante la existencia de embolismos sépticos, sobre todo a nivel pulmonar, habiendo descartado previamente la existencia de lesión endocárdica, sin que sea necesaria la existencia de foco séptico orofaríngeo, y sirviendo el aislamiento en cultivo de anaerobiosis junto a la confirmación de tromboflebitis yugular por técnicas de imagen parea corroborar el diagnóstico, aunque no necesariamente, ya que éste es esencialmente clínico $(2,40)$.

\section{Bibliografía}

1. Lemièrre A. On certain septicemias due to anaerobic organisms. Lancet 1936; 1: 701-3.

2. Garrido JA, Ponte MC, Fernández Guerrero ML. Síndrome de Lemièrre (sepsis postangina): una infección olvidada. Med Clin (Barc) 1989; 93: 660-2.

3. García Ruiz F, Martínez de Antonio E, Sanz Sanz F, García Bravo M. Síndrome de Lemièrre: manifestación tardía de la tromboflebitis yugular interna [carta]. Med Clin (Barc) 1990; 94: 797.

4. Lusting LR, Cusick BC, Cheung SW, Lee KC. Lemièrre`s syndrome: two cases of postanginal sepsis. Otolaryngol Head Neck Surg 1995; 112: 767-72.

5. De Sena S, Rosenfeld DL, Santos S, Keller I. Jugular thrombophlebitis complicating bacterial pharyngitis (Lemièrre`s syndrome). Pediatr Radiol 1996; 141-4.

6. Bielecki JW. Septic syndrome with a pulmonary focus (clinical conference). Schweiz Rundsch Med Prax 1991; 80: 1030-3.

7. Gato A, García Aguado C, Mateo O, Pontes MJ, Baez B, Pérez V. Diagnóstico por imagen en el síndrome de Lemièrre. Enferm Infecc Microbiol Clin 1993; 11: 263-6.

8. Ahkee S, Srinath L, Huang A, Raff MJ, Ramírez JA. Lemièrre`s syndrome: postanginal sepsis due to anaerobic oropharyngeal infection. Ann Otol Rhinol Laryngol 1994; 103: 208-10.

9. Karanas YL, Yim KK, Shuster BA, Lineaweaver WC. Lemièrre`s syndrome: a case of postanginal septicemia and bilateral flank abscesses. Ann Plat Surg 1995; 35: 525-8.

10. Bach MC, Roediger JH, Rinder HM. Septic anaerobic jugular phlebitis with pulmonary embolism: Problems in management. Rev Infect Dis 1988; 10: 424-7.

11. Pulido Rivas P, Masa C, César JM, Garcide Sola R. Asociación de lesiones cerebrales múltiples, trombosis de la yugular y nódulo pulmonar en paciente de 17 años. Rev Clin Esp 1995; 195: 195-7.

12. Weesner CL, Cisek JE. Lemièrre`s syndrome: The forgotten disease. An Emerg Med 1993; 22: 256-8.

13. Martín Joven A, García Román M, Gómez Rodrigo J, Obeso Fernández G. Síndrome de Lemièrre: Sepsis postangina. Rev Clin Esp 1992; 190: 379.

14. Marchan Carranza E, Gijón Rodríguez J, Mantes Germán I. Embolismo pulmonar séptico secundario a foco dentario. ¿Síndrome de Lemièrre? Arch Bronconeumol 1994; 30: 473-4.

15. Kern W, Dolderer M, Krieger D, Buchler M, Kern P. Lemièrre`s syndrome with splenic abscesses. Dtsch Med Wochenschr 1992; 117 1513-7.

16. Goyal M, Sharma R, Jain Y, Gupta A, Berry M. Inusual radiological manifestations of Lemièrre`s syndrome: a case report. Pediatr Radiol 1995; 25 Supl 1: S105-6. 
17. Stahlman GC, DeBoer DK, Green NE. Fusobacterium osteomyelitis and pyarthrosis: a classic case of Lemièrre`s syndrome. J Pediatr Orthop 1996 Jul-Aug; 16: 529-32.

18. Blok WL, Meis JF, Gyssens IC, Gimbrere JS, Horrevorts AM. Postangineuze sepsis door Fusobacterium necrophorum: het syndroom van Lemièrre. Ned Tijdschr Geneeskd 1993; 137: 1013-6.

19. Martínez E, Pardo M, De Diego P. Embolismo pulmonar séptico secundario a un foco de osteítis periodontal. Arch Bronconeumol 1994; 30: 174-5.

20. Lao Luque J, Molina Utrilla R, Lorente Guerrero J, Mateu Vallvardu F, Perello Scherdel E. Síndrome de Lemièrre. A propósito de un caso. An Otorrinolaringol Ibero Am 1993; 20: 599-605.

21. Seidenfeld SM, Sutker WL, Luby JP. Fusobacterium necrophorum septicemia following oropharyngeal infection. JAMA 1982; 248: 1348.

22. Ely EW, Stump TE, Hudspeth AS, Haponik EF. Thoracic complications of dental surgical procedures: hazards of the dental drill (see coments). Am J Med 1993; 95: 456-65.

23. Busch N, Mertens PR, Schonfelder T, Nguyen H, Marschall HU, Kierdorf $\mathrm{H}$, et al. Postangina sepsis Lemièrre mit meningitis und intravasaler verbrauchskoagulophatie als komplikation einer infektiosen mononucleose mit pansinusitis. Dtsch Med Wochenschr 1996; 121: 94-8.

24. Hughes CE, Spear RK, Shinabarger CE, Tuna IC. Septic pulmonary emboly complicating mastoiditis: Lemièrre`s syndrome revisited. Clin Infect Dis 1994; 18: 633-5.

25. Finegold SM. Anaerobic bacteria: General Concepts. En: Mandell GL, Bennet JE, Dolin R, editores. Principles and Practice of infectious Disease, 4th ed. Nueva York: Churchill Livingstone, 1995; 2165-209.

26. Moloy PJ. Anaerobic mastoiditis: a report of two cases with complications. Laryngoscope 1982; 92: 1311-5.

27. Moxley GF, Oqen DS Jr, Irby R. Septic arthritis due to Fusobacterium varium in a patiens with sickle-cell anemia (letter). J Rheumatol 1983; 10: 161-2.

28. Kager L, Nord CE. Imipenem/cilastatin in the treatment of intraabdominal infections: a review of worldwide experience. Rev Infect Dis 1985; 7 Suppl 3: S518-21.

29. Onderdonk AB, Bartlett JG, Louie T, Sullivan Seigler N, Gorbach SL.
Microbial synergy in experimental intra-abdominal abscess. Infect Inmun 1976; 13: 22-6.

30. Brondz I, Olsen I. Multivariate analyses of cellular fatty acids in Bacteroides, Prevotella, Porphyromonas, Wolinella and Campylobacter spp. J Clin Microbiol 1991; 183-9.

31. Olsen I. Chemotaxonomy of Bacteroides: a review. Acta Odontol Scand 1994; 52: 354-67.

32. Brook I. Prevotella and Porphyromona infections in children. J Med Microbiol 1995; 42: 340-7.

33. Fishman EK, Patker RL, Gayler BW, Wheeler PS, Seigelman SS. Yugular vein thrombosis: diagnosis by computed tomography. J Com Assit Tomog 1984; 8: 963-8.

34. Gubler JG, Wuest J, Oneta C, Hany A. Sepsis caused by Fusobacterium necrophorum: the re-discovered postangina sepsis Lemièrre syndrome and other manifestations. Schwerz med Wochenschr 1990; 120: 440-5.

35. Mane S, Torres M, Buges J, Rivas A, Bruno C, Rodríguez E, et al Scientigraphyc demonstration of jugular obstruction in a case of Lemièrre syndrome. Clin Nucl Med 1992; 17: 233-5.

36. Garrido JA, Fernández Fernández FJ, Ameneiros E, Gallego JC. Abdomen agudo como forma de presentación de un síndrome de Lemièrre. Med Clin (Barc) 1995; 105: 36-7.

37. Hara RP, MacDonald A, Pullen D, Ganesan S, Prior AJ. Lemièrre's syndrome: are we underdiagnosing this life-threatening infection?. ORL J Otorhinolaryngol Relat Spec 1996; 58: 178-81.

38. Cosgrove EF, Colodny SM, Pesce RR. Adult respiratory distress syndrome as a complication of postanginal sepsis. Chest 1993; 103: 1628-9.

39. Verghese A, Widrich WC, Arbeit RD. Central Venous septic thrombophlebitis. The role of medical therapy. Medicine 1985; 64: 394-400.

40. Ruiz JM, Castillo C, Manzano A, Corral E. Sepsis postangina. Rev Clin Esp 1993; 193: 45

41. Sinave CP, Hardy GJ, Fardy PW. The Lemièrre`s syndrome: Suppurative thrombophlebitis of the internal jugular vein secondary to oropharyngeal infection. Medicine 1989; 68: 85-94.

42. Celikel TH, Muthuswamy PP. Sptic pulmonary emboli secondary to internal jugular vein phlebitis (postanginal sepsis) caused by Eikenella corrodens. Am Rev Respir Dis 1984; 130: 510-3. 